CONF-901275-.1

UCRL - JC-105999

PREPRINT

\title{
LINE AND CONTINUUM SPECTROSCOPY AS DIAGNOSTIC TOOLS FOR GAMMA RAY BURSTS
}

\author{
Edison P. Liang \\ Lawrence Livermore National Laboratory \\ University of Califomia \\ Livermore, California 94550
}

This paper was prepared for submittal to the proceedings of the

Gamma-Ray Line Astrophysics Symposium

December 10-13, 1990

Paris-Saclay

December, 1990

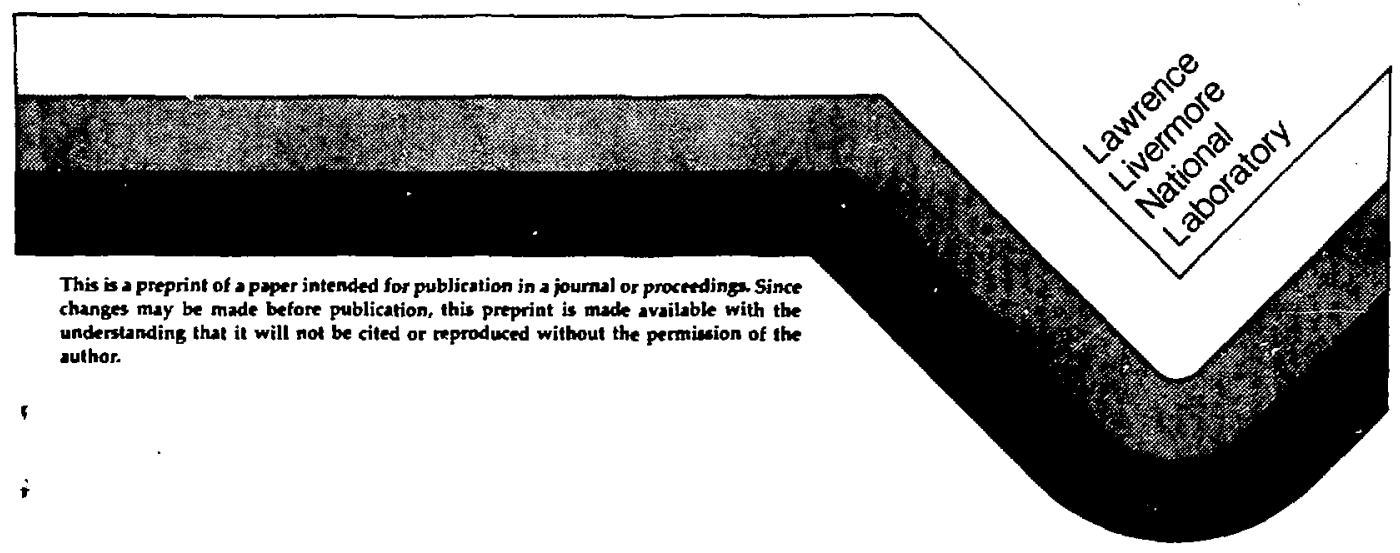




\section{DISCTAIMER}

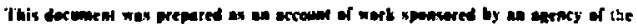

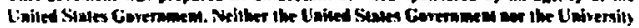

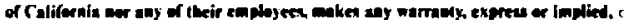

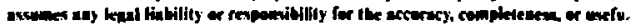

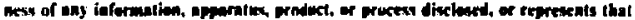

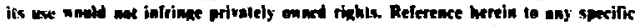

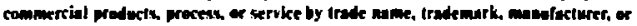

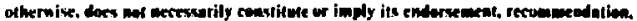

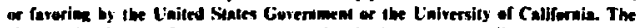

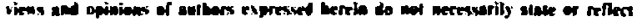

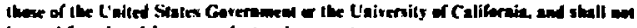

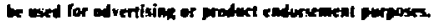

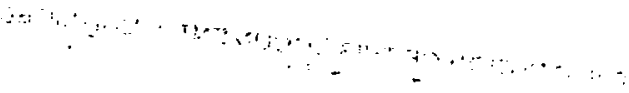




\title{
LINE AND CONTINUUM SPECTROSCOPY AS DIAGNOSTIC TOOLS FOR GAMMA RAY BURSTS
}

\author{
Edison P. Liang \\ LLNL, University of Califomia \\ Livermore, CA 94550
}

\begin{abstract}
We review the theoretical framework of both line and continuum spectra formation in gamma ray bursts. These include the cyclotron features at 10 's of keV, redshifted annihilation features at $\sim 400 \mathrm{keV}$, as well as other potentially detectable nuclear transition lines, atomic X-ray lines, proton cyclotron lines and plasma oscillation lines. By combining the parameters derived from line and continuum modeling we can try to reconstruct the location, geometry and physical conditions of the burst emission region, thereby constraining and discriminating the astrophysical models. Hence spectroscopy with current and future generations of detectors should provide powerful diagnostic tools for gamma ray bursters.
\end{abstract}

\section{INTRODUCTION}

Study of spectral lines has been the backbone of astrophysics. It provides us with critical informations about the physical conditions of remote environments and exotic phenomena. Line spectroscopy is the standard diagnostic tool of objects from stars to galaxies. Though this is a symposium on gamma ray lines, in the case of gamma ray bursts (GRBs), it is not possible to discuss the study of spectral lines without first addressing the issues related to the formation of the continuum, hence the title of this review talk. Since the bulk of the observational results is already reviewed in Vedrenne's talk (this volume), I will concentrate on the theoretical issues, reviewing the observational facts only briefly when necessary.

Adopting a somewhat biased approach, I will consider the highest near-term priority in the study of gamma ray bursts to be the verification of the strong field $\left(B \sim 10^{12} G\right)$ Galactic isolated neutron star hypothesis. If this TGNS (teragauss Galactic neutron star) paradigm can indeed be proven beyond doubt, then the next goal of gamma ray burst spectroscopy would be to diagnose the detailed geometry and physical conditions of the emission and line forming regions. This will in turn constrain the various astrophysical and energy source models, ranging from star quakes to cometary impacts. Beyond that the study of GRB as the most populous species of Galactic neutron sters will tell us much about neutron star origin and evolution (e.g. Hartmann, this volume), and perhaps even the structure and evolution of the Galaxy as a whole. But first we must consolidate the evidences for the TGNS picture and resolve all of its remaining puzzles and difficulties. Hence in this talk I will concentrate on the bearing of line and continuum spectroscopy on the TGNS picture. Recent reviews of GRB spectra can be found in Harding (1991), Hurley and Lamb (1991), Higdon and Lingenfelter (1990), Liang (1989) and the monographs edited by Ho and Epstein (1991) and Liang and Petrosian (1986).

\section{TIME-AVERAGED SPECTRA}

Much of our interpretations of GRBs come from their time-averaged spectra, with integration times ranging from -fraction of a second in SIGNE and PROGNOZ experiments (Atteia et al. 1987) to 16 seconds for SMM (Nolan et al. 1984, Share et al. 1986). But the true spectral evolution time scale is likely much shorter, say typically $<10 \mathrm{~ms}$, especially for narrow spectral features (e.g. GB790305). Hence when we discuss the generic GRB spectra using timeaveraged samples, we should keep in mind that these may not be truly representative of the physical condition on shori time scales. 
Fig. 1 reproduces the generic GRB spectrum. It consists of an exponential shape from 10 's of $\mathrm{keV}$ to $-\mathrm{MeV}$ followed by a power law tail above an MeV of photon index 2 - 3. At least for a few events this tail extends out to $100 \mathrm{MeV}$ and GRO stould be able to see if it goes much higher. The continuum typically breaks to a slope of flatter than index 2 at a few hundred keV, continuing to flatten towards lower energies. In cases where $\mathrm{x}$-rays are detected the $\mathrm{x}$-ray spectrum below $10 \mathrm{keV}$ seems to be consistent with a simple extrapolation of the gamma ray spectrum, leading to a $x / g a m m a$ ray luminosity ratio of $<2 \%$. However, the $x$-rays seem to persist long after the gamma rays die off, and in a few instances there is also an $x$-ray precursor (Murakami 1991). The spectrum of the $x$-ray tail and the precursor is much softer, consistent with a blackbody of $\leq 2 \mathrm{keV}$. Hence it likely represents a different emission component. In addition to $X$-rays, archival optical transierits have been detected in several GRB error boxes (Schaefer 1981) which may correspona to $-10^{-3}$ of the flux of the gamma rays. However, recent works on plate defects cast doubts on the meaning of the optical images and we can only conservatively put upper limits on the optical flux. Similarly, there are only up limits on the emission of other low frequency radiation from radio to infrared (Fig. 2).

In 10-25\% of the events the spectra show evidence of time-dependent line features. Recent GINGA confirmation of multi-harmonic absorption dips at $\sim 20-40 \mathrm{keV}$ (Murakami et al. 1988) gave credence to the interpretation of these features as cyclotron harmonics in a teragauss magnetic field, first observed in KONUS data (Mazets et al. 1980). Less certain are the alleged redshifted annihilation features at $\sim 400 \mathrm{keV}$ reported by KONUS, which was not confirmed by SMM observations. The reality of these features must await confirmation by GRANAT or GRO, but it is at least worth discussing here their implications if they are real. In at least une event (GB781119) there was evidence of additional line features at $740 \mathrm{keV}$ and higher energies, which may be interpreted as nuclear lines. So far there has been no observation of atomic lines in the $x$-ray range, either in the main part of the burst or in the $x$-ray tail or precursor. But future $x$-ray observations with HETE and other experiments may change that.

\section{SPECTRAL EVOLUTION}

Almost all observations to date suggest that the spectral features, whether it is the cyclotron features at 10 's of $\mathrm{keV}$ or annihilation features at $-400 \mathrm{keV}$, vary with time during a single burst. Fig. 3 illustrates the spectral evolution of GB880205 observed by GINGA showing that the double cyclotron features appear most prominent only during the brief middle part of the spike. More recent sudies (Murakami 1991) suggest that the line center position also seem to evolve downward even within that time interval. As for the annihilation line, the most celebrated example is that of GB790305, which may be present only during the first 24 milliseconds of the burst (Barat et al. 1983).

The continuum itself shows evidence of rapid evolution down to 10's of ms (Mitrofanov 1991). Study by Norris et al. (1986) and other groups suggest that the spectral hardness deaks before total count rate and the overall evolution of each spike is from hard to soft (I ig.4). Golenetstii et al. (1984) on the other hand claimed that there is a close correlation between luminosity and spectral hardness. Despite its controversy this result has recently been confirmed by the study of SIGNE data (Liang et al. 1991, Fig.5). It is found that the luminosity is tightly correlated with the best-fit bremsstrahlung temperature according to $L \sim \mathrm{T}^{1} .65 \pm .28$ down to 0.5 sec. Note that this result need not conflict with that of Norris et al. (1986) since luminosity is proportional to count rate times temperature $T$. So even though the rising phase of the burst has fewer counts its luminosity may still be high due to the hardness of the photons. If confirmed this result has interesting implications for the burst mechanism. 


\section{THE TGNS PARADIGM}

Fig.6 illustrates the standard arguments in favor of a teragauss field neutron star origin of gamma ray bursts. Millisecond and submillisecond time variability constrains the size of the emission region to that of 10 's to 100 's of $\mathrm{km}$. Cyclotron lines at 10 's of keV show that at least the emission region of the soft gamma rays below $100 \mathrm{keV}$ (not necessary the gamma rays above several $\mathrm{MeV}$ ) must come from very strong field regions. If the annihilation line interpretation of the $400 \mathrm{keV}$ features is indeed correct and the shift of line-center position is all due to gravitational redshift, then it is both a strong confirmation of the neutron star origin and a constraint on the neutron star structure. So far the only periods from GRBs that seem convincing are the 8 second period of GB790305 and the 2.2 second period of two recent bursts (Kouveliotou et al. 1988, Owens et al. 1990). But if future observations can firmly establish the existence of periods for more bursts, either from the gamma ray light curve or the longer $x$-ray tail, then it is another evidence in favor of strong fields.

Other arguments in favor of a strong magnetic field include the efficient acceleration of energetic ( $>100 \mathrm{MeV}$ ) particles which is facilitated by the presence of ultrastrong fields, and the confinement of the radiating plasmas which would have expanded at the speed of light due to thermal and radiation pressure. None of the above arguments is watertight, of course, and there are always more counter examples than examples. But at this point a TGNS picture seems to be the most useful baseline framework from which we can build meaningful models and confront them with current and future observational data. However, it should be emphasized that even if the TGNS paradigm turns out to be correct for a large majority of classical GRBs, this does not exclude the existence of subclasses of GRBs (e.g. soft repeaters, short soft bursts etc) that have other origins.

The TGNS paradigm comes with many puzzles, both physical and astronomical. We list some of them here:

1. Why are periods so rare?

2. Why are there no attenuation of multi-MeV gamma rays from gamma-gamma and gamma-B pair production?

3. Why are there so few $x$-rays?

4. Why are the cyclotron lines so narrow if the underlying continuum is so hot?

5. If GRBs derive from old, isolated neutron stars past their pulsar phase, why are their fields so strong?

6. Why are cyclotron and annihilation features so common (if we believe KONUS statistics) in GRBs but so rare in radio pulsars?

7. Why is there no radio emission from GRBs?

8. If the blackbody interpretation of the $x$-ray tail is correct, then it is consistent with a typical source distance of $\geq$ kpc unless only a tiny fraction of the stellar surface is emitting. How can this be reconciled with the isotropy, $V / V_{\max }$ and $\log \mathrm{N}-\log \mathrm{P}$ results if the sources are Galactic neutron stars?

\section{CONTINUUM DLAGNOSTICS}

The interpretation of any spectral line observed in GRB spectra would depend on the model for the continuum. Hence we must first address the origin of the continuum spectrum, say from 
$\sim$ keV out to $\geq 100 \mathrm{MeV}$. At present there are at least two competing scenarios concerning the origin of the continuum emission: (a) surface or near-surface emission from beamed relativistic electrons (and pairs) for the entire spectrum, and (b) two component unbeamed emission with the sub-MeV soft component coming from near the surface and the super-MeV power-law tail coming from high in the magnetosphere away from the surface. Artist conceptions of these scenarios are depicted in Fig.7. The fundamental constraints that force us into one of these two scenarios are: a) lack of $x$-rays so that reprocessing from the stellar surface must be minimal; hence either the emitting particles are beamed away from the surface, or the primary emitting region must be far enough away from the surface so that only a small fraction of the energy release reaches the surface; b) lack of gamma-gamma or gamma-B e+e-pair production attenuation of the super-MeV gamma rays; this means that either the high energy gamma rays are emitted parallel to the field lines, or the field has to be weak enough ( $510^{1}{ }_{G}$ for isotropic photons). At this point both scenarios are still viable. But future high time resolution correlation study (say down to $10 \mu \mathrm{s}$ ) of the two spectral components should easily discriminate between them since in the former case both components are emitted simultaneously, at the same location and by the same bunch of particles, whereas in the latter case the soft component should lag the hard one by at least the light travel time. These and other consequences of the two scenarios and their implications for the spectral interpretations are summarized in Table 1.

The most advanced version of the former scenario is called the cyclotron or resonance upscantering process (CUSP, Hameury et al. 1985, Dermer 1989, Ho and Epstein 1989, 1991, Vitello and Dermer 1991): thermal ( $\mathrm{seV}$ ) soft photons emitted by the stellar surface are upscattered with the resonance cross section (-hundreds times Thomson) by outwarding streaming relativistic electrons or pairs (Lorentz factor $\gg$ i) into forward-beamed gamma ray photons. To the extent that the pairs are injected with a power law, the output optically thin gamma spectrum will have a power low at high energies and break to a flat spectrum (photon index 0 ) if the photon target is thin and break to spectrum of index 1 if the photon target is thick. The break energy is given by $\sim v_{\mathrm{B}}^{2} / v_{0}$ in the former case where $v_{\mathrm{B}}$ is cyclotron energy and by $\gamma_{*} v_{\mathrm{B}}$ where $\gamma_{*}$ is the low energy cutoff of the electron distribution in the latter case. Hence the most critical feature of this model is tha existence of the break energy winich is a measure of the soft photon temperature or the electron distribution cutoff. Note that if the spectral index below the break is flatter than 1 the photons must be thin target whereas if it is steeper than 1 it could be either thin or thick target because in that case the low energy component must be synthesized by superposition of spectra of different break energies. In most models the output spectrum will also show a soft $x$-ray bump corresponding to the unscattered input spectrum but in reality this may be washed out by optical depth effects. To summarize, in the coniext of the CUSP model the key spectral parameters are: break energy; spectral indices above and below the break and the location of the soft photon peak, if any. The emission parameters to be extracted from spectral fitting with this model are: electron injection index and low energy cutoff, soft photon temperature, B-field strength (from lines if detectable) and electron Thomson depth.

In the two component model the spectral break would be interpreted as the low energy cutoff of the energetic electrons emitring in the magnetosphere. The soft component below the break will be either reprocessed radiation or secondary emission with energy supplied directly to the surface (e.g by Alfven waves or particle flux if the primary energy source is external or by thermal diffusion if the primary energy source is internal). While most authors (e.g. Ruderman 1987, Liang 1987, Sturrock 1986, Harding and Sturrock 1989, Peirosian 1991) visualize the farfield high energy component to be some form of $E_{/ /}$-induced pair-cascade (from curvature, synchrotron and inverse Compton processes) due to the similarity of the index to those of pulars 
$(-2-2.5)$, there is as yet no concensus as to the origin of the low-energy component. The only constraint is that it originates near the strong-field stellar surface in cases we see cyclotron absorption or redshifted annihilation features. It could be emitted by a combination of resonant scattering and synchrotron, or even bremsstrahlung and nonmagnetic Compton in cases where there is no evidence of strong field (e.g. B $<5 \times 10^{10} \mathrm{G}$ ). The emitting particles could be Maxwellian (due to collisionless relaxations) or nonMaxwellian (e.g. cooling) distributions. If the distribution of the low-energy electrons is indeed Maxwellian then the overall curvature of the lowenergy component usually measured by bremsstrahlung fits will give a characterization of the mean electron energy. If the spectrum further turns over in the $x$-rays as hinted by some bursts (e.g. GB811016 Katoh et al. 1984 and GB880205) then the turnover energy also constrains the emission parameters. If the turnover is due to self-absorption or saturation of scattering we derive the emission measure or Thomson depth modulo temperature and field strength (if cyclotron line detected). If the turnover is due to first harmonic cutoff (and no line detected above turnover) we derive the field strength. If the turnover is due to plasma frequency cutoff (Razin-Tystovich effect) we derive the electron (pair) density. If the turnover is due to absorption by circumstellar or interstellar matter we derive the optical depth and composition of intervening matter, etc. Discrimination of these and other models can only be achieved by refined modeling and imevariability analyses.

In summary, continuum spectral measurements will give us a handle on a number of emission parameters. Interpretation of the high energy power-law component seems to be least sensitive to the choice of model or scenario, giving us unambiguous estimate of the electron index and low-energy cutoff. But parameters derived using the low energy component below a few hundred $\mathrm{keV}$ is sensitive to the specific emission model we choose. Discrimination among these models must await further study, including rapid time-variability correlation analyses.

\section{LINE DIAGNOSTICS}

Line features, when available, will give us critical informations which supplement the continuum parameters. However, their interpretation is again scenario dependent and the physical parameter derived in most cases is sensitive to the model adopted. In addition to cyclotron features at 10 's of $\mathrm{keV}$ and annihilation features at $\sim 400 \mathrm{keV}$, whose detections have some credibility, we will also give more speculative discussions of other possible lines and their use as diagnostic tools.

A. Cyclotron Features

Since their first discovery by KONUS (Mazets et al. 1980, Golenetskii et al. 1986) the reality or interpretation of these feanures have remained controversial until their recent confirmation by GINGA (Mumakami et al. 1988) with the discovery of multiple harmonics. The fundamental difficulty, of course, is observational uncertainty due to nonuniqueness or obligeness of the decorvolution procedure of count spectra to photon spectra. Even when one accepts the features as real there is still room for speculation as to their origin (e.g superposition of multicomponent continuua, Lasota and Belli 1983, K-edge absorption by heavy elements, Bisnovaty-Kogan 1987, synchotron emission instead of absorption, Brainerd and Lamb 1987, broad emission dip with line-centr: reversal, Fenimore et al. 1988 etc.). But after many man-hours of detailed modeling, at this point by far the most popular and satisfactory interpretation is that these cyclotron features are produced by resonant scattering by cool electrons $\left(T_{/ /} \leq 5 \mathrm{keV}\right)$ in which photon-spawning from second harmonic decay partially fills in the first harmonic, producing the apparent strength of the second harmonic versus the first. Analyses invoking both harmonics (or maybe even the third) seem to give a scatering depth of $10^{21} \cdot 10^{22} \mathrm{~cm}^{-2}$ depending on the geometry, dynamics and view angle (Wang et al. 1989, Alexancier and Meszaros 1989). The unambiguous parameter is the field 
strength, which is -2 teragauss modulo any redshift correction. The narrowness of the line constrains both the absorbing medium temperature $(T / / \leq 5 \mathrm{keV})$ and field gradient $(\Delta \mathrm{B} / \mathrm{B} \leq 30 \%)$. But at this point the absorption site, geometry and dynamics (wind or static medium) are all unknown.

Detection of absorption features means that at other view angles at other times, we shuuld have a shance of seeing emission features. In adidition to the narrow emission features predicted to come out from the cool scattering layer (Wang et al. 1989), broad emission features could also arise as part of the continuum when it is due to synchrotron emission if particles are energetized to high Landau levels (Fig.8, Brainerd and Lamb 1987). In addition to a measure of the field strength, such emission features would help to constrain the emission geometry and give consistency check of the continuum emission model. Table 2 summarizes the use of cyclotron features as diagnostic tools.

\section{B. Annihilation Features}

Besides the celebrated March 5 event (CB790305), there are only a few other widely recognized narrow annihilation feature in classical GRBs observed by several independent experiments, notably GB781119 (Fig. 9) (see Harjing et al. 1986 for review). Besides the KONUS results which show annihilation features in a significant fraction of the bursts (Mazets et al. 1980) a good example of a broad feature at $\sim 400 \mathrm{keV}$ can also be found in the HEAO-A4 data (Fig. 10, Heuter 1987). Independent of the ultimate validity of such claims, it is meaningful to examine the implication of such line features and their potential as diagnostic tools.

The first important feature of the annihilation lines is that they appear to be redshifted. Liang (1986) analyzed the data of almost 40 events with apparent features and conciuded that the redshift database is most consistent with a medium-sofi neutron star equation of state. However this analysis is beset with caveats and uncertainties (Kluzinak 1989). Many factors can contribute to the shifting of the line centroid besides gravity. These include thermal, bulk Doppler (orbital or turbulent motion), Compton scattering, opacity effects, strong magnetic fields etc. Despite these uncertainties, future redshift measurements of GRB annihilation lines will remain the most powerful tool for the stidy of neutron star structure and relativistic effects.

Besides the line center position, two other observable parameters are the line width and line intensity. Like any other line diagnostics, these would provide constraints on the pair density. Thomson depth, field strength, temperature (of both the annihilating medium and any intervening scatterer), etc. For example, if the field is approaching the critical field of $4.4 \times 10^{13} \mathrm{G}$, then the annihilation would be dominated by second harmonic (1-photon) emission at $1022 \mathrm{keV}$ and the profile would be strongly asymmetric (Harding 1986). Ratio of the line to integrated power-law continuum intensity can potentially discriminate between the pair cascade and other models (Sturrock 1986). If the shift is due to Compton down-scattering, then the line center position is related to the width. Table 3 summarizes the implications of the $511 \mathrm{keV}$ line.

\section{Other Lines}

In addition to the annihilation feature, GB781119 (Fig.9) also shows evidence of a line at $740 \mathrm{keV}$ and weaker features at higher energies. This could be inierpreted as redshifted $870 \mathrm{keV}$ Fe deexcitation line. Given the high temperature and high gamma intensity of the source it is quite plausible, or even likely that Fe nuclei, if aburdant, would be excited, and the $870 \mathrm{keV}$ and other Fe deexcitation lines (and those of other nuclei, including the $2.2 \mathrm{MeV}$ line of D and $4.4 \mathrm{MeV}$ line of $\mathrm{C}$ ) would provide interesting diagnostics of the source. But we must first await the result of GRO and other future experiments. 
So far no line feature has yet been discovered in the soft $x$-ray regime below $10 \mathrm{keV}$, primarily due to the lack of $\mathrm{x}$-ray events and poor instrumental resolution. But if $\mathrm{Fe}$ is the abundant element in the burst environment, then at least during the precursor and $x$-ray tail when the temperature is cooler ( $\leq 2 \mathrm{keV}$ from GIN(FA), detection of Fe lines in the $6-7 \mathrm{keV}$ range as well as spectral jump at the K-edge should be quite likely. Together these should provide a wealth of diagnostic tools for the $x$-ray emission region. Of course the $x$-ray emission region may be totally separate and different from the gamma ray emission region, but at least we will have some informations on the underlying neutron star environment, such as $\mathrm{Fe}$ abundance and redshift, winds or accretion flows. Just as Fe K-lines are expected to provide powerful diagnostic tools from X-ray binaries to clusters of galaxies (e.g Holt and McCray 1982), we anticipate Fe K-lines will also be a useful diagnostic for GRBs.

The acceleration of energetic particles responsible for the high energy continuum will likely lead to excitation of a variety of plasma instabilities either as a cause or consequence (c.g. Melrose 1980), most of which will end up dumping some of their energies into longitudinal electron plasma oscillations (Langmuir waves). A by-product of such excitations is the emission of plasma line radiation at $\omega_{e}$ (plasma frequency) and $2 \omega_{e}$ (Liang 1985), as in the case of solar radio bursts. At present the plasma density of the emission region is unknown, but if it lies in the range $10^{18}$. $10^{24} \mathrm{~cm}^{-3}$ deduced from various considerations (see e.g. Liang 1985), then the plasma frequency ( $=0.4 \mathrm{eV}\left(\mathrm{n} / 10^{20}\right)^{1 / 2}$ ) would lie in the IR to XUV range. Future optical-UV monitoring of GRB (c.g. with HETE) might have a chance of detecting this.

Another line that may lie in the UV range is that of ion cyclotron emission or absorption (Kirk and Stoneham 1982) in a $10^{12} \mathrm{G}$ field. Since the opacity is much lower than that of Thomson it might be difficult to detect. Table 4 summarizes the results for these other lines.

\section{CONCLUSHONS AND SUMMARY}

Line and Continuum spectroscopy of GRBs is expected to provide powerful diagnostic tools of the emission and absomtion regions. Within the context of a specific model or scenario, line and continuum data should be able to provide us with rich informations about the emission site, geometry, and physical par meters such as density, temperature or electron distribution, optical depth, magnetic field strength, bulk motion etc. Time evolution of the spectra will give forther informations about the dynamics and energetics of the source. With the observations of GRANAT, GRO, ULYSSES, HETE and other missions we have high hopes that spectroscopic diagnostic of gamma ray bursts will become a reality. This will allow us to not only clarify the astronomical and astrophysical issues of GRBs, but also use GRBs as laboratories for the study of strong-field, relativistic plasma physics found nowhere else in the universe.

\section{ACKNOWLEDGEMENTS}

This work was performed under the auspices of the U.S. Department of Energy by the Lawrence Livermore National Laboratory under contract number W-7405-ENG-48.

The author thanks C.D. Dermer and D. Hartmann for helpful conversations and the conference organizers for partial support. 


\section{REFERENCES}

Alexander, S. G. and Meszares, P., 1989, Ap. J. Lett. 344, L1.

Atteia, J. L. et al. 1987, Ap. J. Supp. 64, 305.

Barat, C. et al. 1983, in AIP Conf. No. 101, ed. M. Burns, A. Harding and R. Ramaty (AIP, NY).

Bisnovaty-Kogan, G. S., 1987, in Proc. LAO Symp. 125, ed. D. Helfand (Reidel, Holland).

Brianerd, J. and Lamb, D. Q. 1987, Ap. J. 313, 231.

Dermer, C. D. 1989, Ap. J. 360, 197.

Fenimore, E. et al. 1988, Ap. J. Lett. 335, L71.

Golenetskii, S. et al. 1984, Nature 307, 41.

Golenetskii, S. et al. 1986, Ap. Sp. Sci. 124, 243.

Hameury, J. M., 1985, Ap. J. 239, 56.

Harding, A. K. 1986, Ap. J. 300, 167.

Harding, A. K. et al. 1986, Ch. 2 in AIP Conf. Proc. No. 141, ed. E. Liang and V. Petrosian, p. 75 (AIP, NY).

Harding, A. K. 1991, Physics Reports to appear.

Harding, A. K. and Snurrock, P. 1989, Ap. J. to appear.

Higdon, J. and Lingenfelter, R., 1990, Ann. Rev. Ast. Ap. 28, 401.

Ho, C. and Epstein, R., 1989, Ap. J. 343, 277.

Ho, C. and Epstein, R. 1991, in Proc. of Taos Workshop on Gamma Ray Bursts, ed. Ho. C. and Epstein R. (AIP, NY).

Holt, S. and McCray, R. 1982, Ann. Rev. Ast. Ap. 20, 323.

Hueter, G. J., 1987, Ph.D. Thesis, U.C. San Diego, pp. 304.

Hurley, K. and Lamb D. Q. 1991, Physics Reports to appear.

Katoh, et al. 1984, in AIP Conf. Proc. No. 115, p. 390, ed. S. Woosley (AIP, NY).

Kirk, J. and Stoneham, R. J., 1982, MNRAS. 201, 1183

Kluzniak, W. 1989, Ap. J. 336, 367.

Kouveliotou, C. et al. 1988, Ap. J. Lett. 330, L101.

Lamb, D. Q. et al. 1990, Ap. J. 363, 670.

Lasota, J. and Belli, 1983, Nature 304, 139.

Liang, E. P. 1985, Nature 313, 202.

Liang, E. P. 1986, Ap. J. 304, 682.

Liang, E. P. 1987, Comín. Ap. 12, 35.

Liang, E. P., 1989, GRO Science Workshop, Proc. p. 4-397, ed. W. N. Johnson (NASA).

Liang, E. P. et al. 1991, unpublished.

Liang, E. P. and Petrosian, V., 1986, editors, AIP Conf. Proc. No. 141 (AIP, NY).

Mazets, E. P. et al. 1980, Ap. Sp. Sci. 80, 3. 
Melrose, D. 1980, Plasma Astrophys. (Gordon \& Breach, NY).

Mitrofanov, I. G. 1991 in Proc. Taos Workshop on Gamma Ray Bursts, ed. C.Ho and R. Epstein (AIP, NY).

Murakami, T. ot al. 1988, Nature 335, 234.

Murakami, T. 1991, in Proc. of Taos Workshop on Gamma Ray Bursts, ed. C. Ho and R. Epstein (AIP, NY).

Nolan, P. et al. 1984, Nature 311, 360.

Norris, J. et al. 1986, Ap. J. 301, 213.

Owens, A. et al. 1990, Ap. J. 352, 741.

Petrosian, V. 1991 in Proc. Taos Workshop on Gamma Ray Bursts, ed. C. Ho and R. Epstein (AIP, NY).

Ruderman, M. 1987 in Cargese NATO ASI Proc., ed. F. Pacini (Reidel, Holland).

Schaefer, B. 1981, Nature 294, 722.

Shore, G. et al. 1986, Adv. Sp. Res. 6 (4), 15.

Sturrock, P. 1986, Nature, 321, 47.

Teegarden, B. J. and Cline, T. L., 1980, Ap. J. Lett. 236, L57.

Virello, P. and Dermer, C. D. 1991, Ap. J. submitted.

Wang, J. C. L. et al. 1989, Phys. Rev. Lett. 63, 1550. 
Table 1: Comparison of Two GRB Continuum Emission Scenarios

Single-Compo:ent Near-Surface Emission entire spectrum former near surface

particles and gamma rays outwardly beamed // local $\mathrm{B}$

no time delay between hard and soft

requires soft $(<\mathrm{KeV}) \mathrm{x}$-ray source

predicts outflowing wind

prefers open flux tubes

spectral hardiness strong function of view angle

break energy determined by field strength and soft photon temperature

primary energy source likely interior

emission region density likely high

E// generation more difficuli
Two-Compinent Ëmission $>\mathrm{MeV}$ tail emiued at $\mathrm{h} \gg 10 \mathrm{~km}$ $<100 \mathrm{KeV}$ emitted near surface uncrnstrained

soft lags hard by $>\mathrm{h} / \mathrm{c}>>10$ Hs not required not predicted preters closed flux tubes insensitive to view angle break energy characuctizes electron energy only can be interior or exterior density likely low E/generation casier

Table 2: Diagnostics with Cyclotron Lines

\begin{tabular}{|l|l|}
\multicolumn{1}{c}{ Measured } & \multicolumn{1}{c|}{ Derived } \\
\hline line center & B-field (module redshift, bulk Doppler) \\
\hline line width & T of scatterer, $\Delta \mathrm{B} / \mathrm{B}$ \\
\hline equivalent width (EW) & Thomson depth of scatterer \\
\hline relative strength of hamnonics & view angle, geometry \\
\hline
\end{tabular}

Table 3: Diagnostics with Annihilation Lines

Measured

\begin{tabular}{|l|l|}
\multicolumn{1}{c|}{ Measured } & \multicolumn{1}{c|}{ Derived } \\
\hline line center & gravitarional redshift (module bulk Doppler) \\
\hline EW & T of pairs and scatterer \\
\hline profile asymmetry & pair density \\
\hline relative strength of harmonics (511/1022) & compactness, B-field (if close to B Brii) \\
\hline ratio of line to continuum & B-field, view angle \\
\hline if redshiff line width & iniection Lorentz factor of pairs \\
\hline if blueshift line width & hint of Compton downscattering \\
\hline
\end{tabular}

Table 4: Diagnostirs with Other Lines

Type of Line

\begin{tabular}{|l|}
\hline nuclear deexcitation lines \\
\hline Fe x-ray lines \\
\hline plasma oscillation lines \\
\hline ion cyclotron lines \\
\hline
\end{tabular}

Derived Parameters abundance, $T_{\text {ion, }}$ redshift abundance, $T_{e}$, redshift, bulk Doppler, ionization states elect on density, $T_{e}$ B-field, $T_{i 00}$ ion Thomson depth 


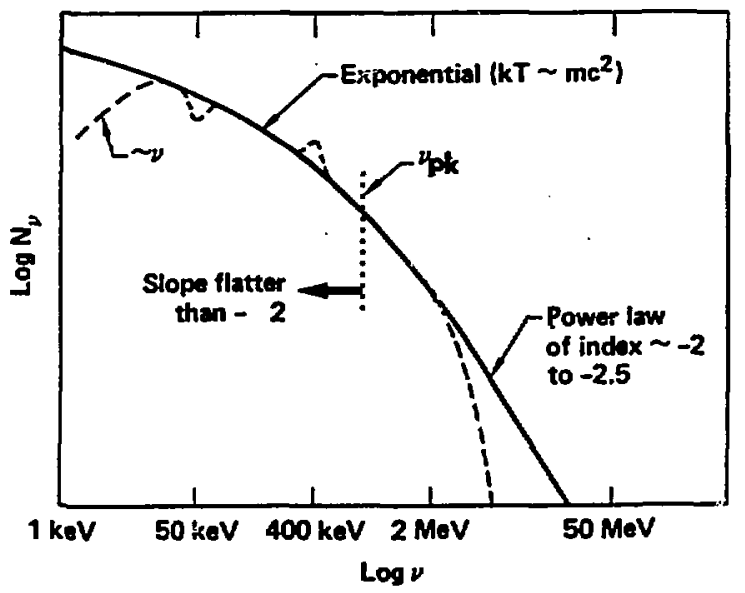

Fig. 1 Generic GRB spectrum. Dashed features appear only in a fraction of observed spectra (from Liang 1987)

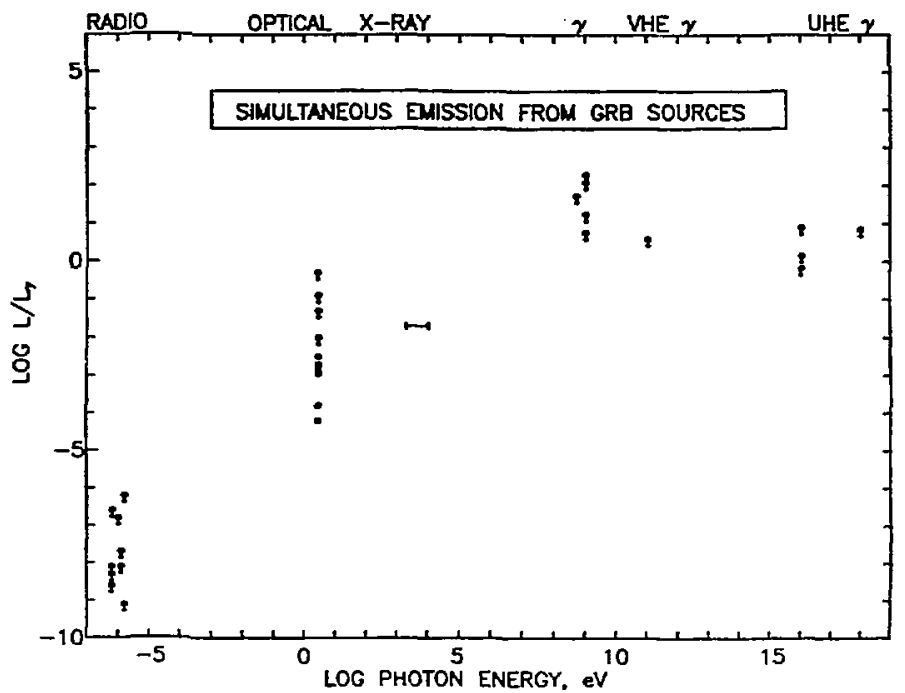

Fig. 2 Limits on GRB continuum emissions from radio to $>100 \mathrm{MeV}$ (from Hurley, 1991) 

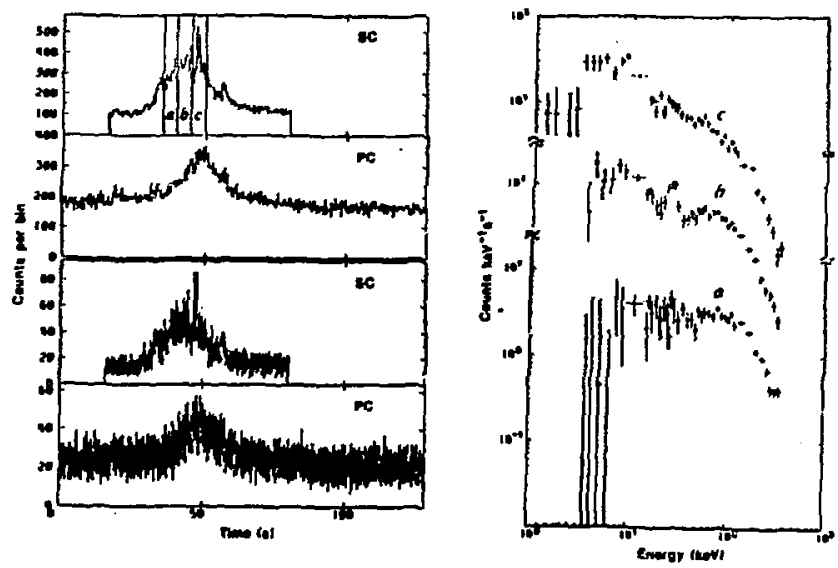

Fig. 3 Time profile and spectra of GB880205 observed by GNGA (from Murakami et al 1988)
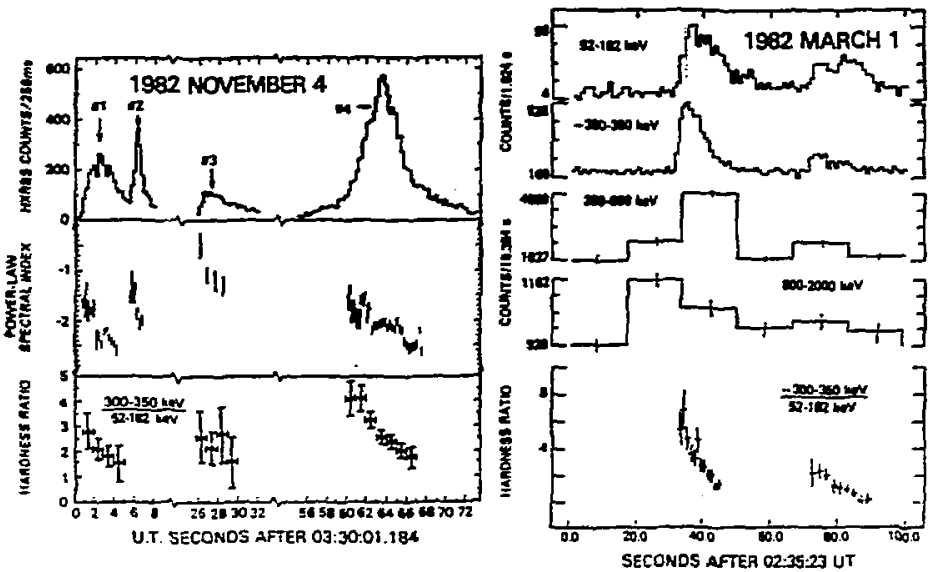

Fig. 4 Evolution of hardness ratio vs. burst time histery for GB821 104 (from Norris et al 1986) 


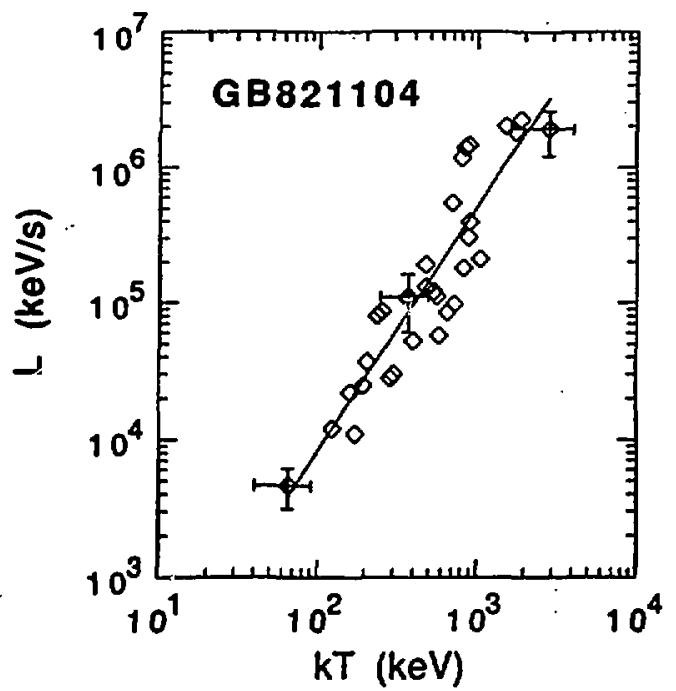

Fig. 5 Preliminary results of luminosity-temperaturs correlation based on 0.5 sec spectral fitting with bremsstrahlung model (Liang et al 1991, unpublished)

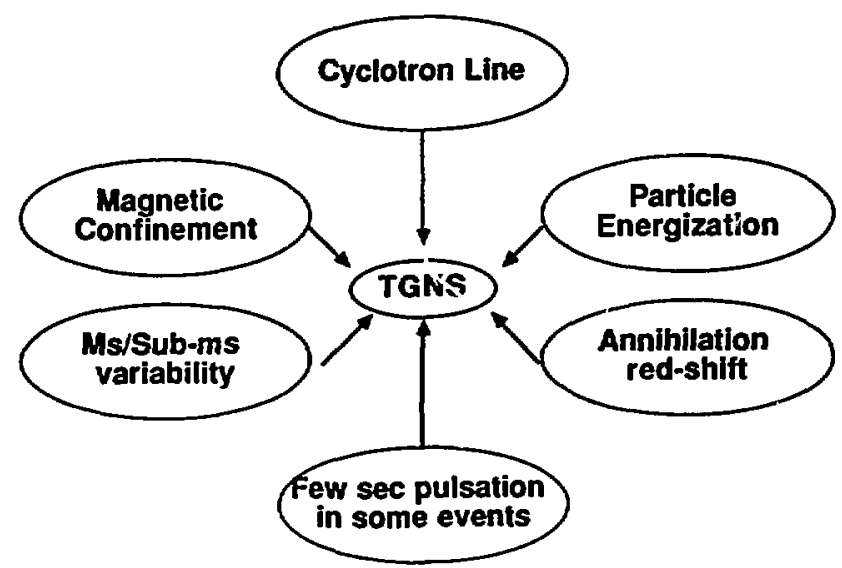

Fig. 6 Support for the TGNS paradigm 

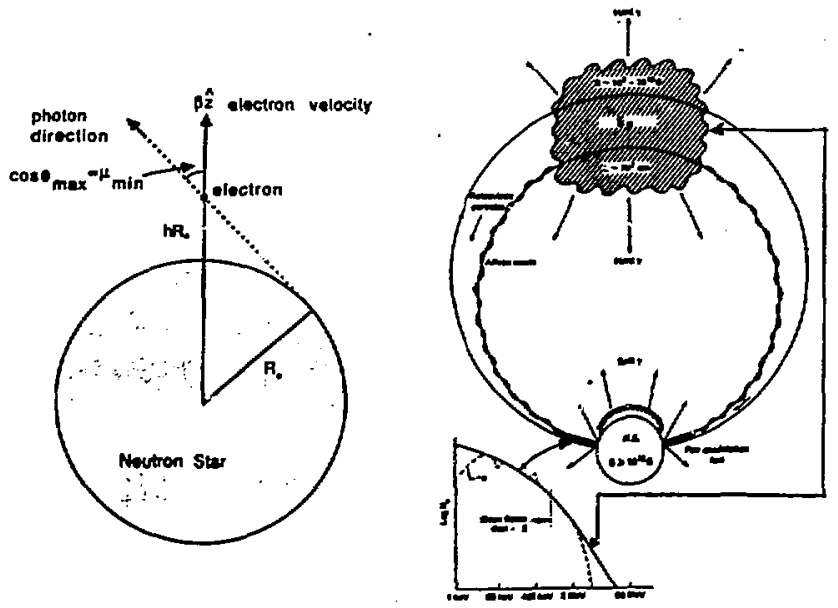

Fig. 7 Artist conception of two different scenarios of continuum spectra formation: (a) emissions by outwardly beamed electrons (pairs) near stellar surface (from Ho and Epstein 1989); (b) two component emission (from Liang 1989)
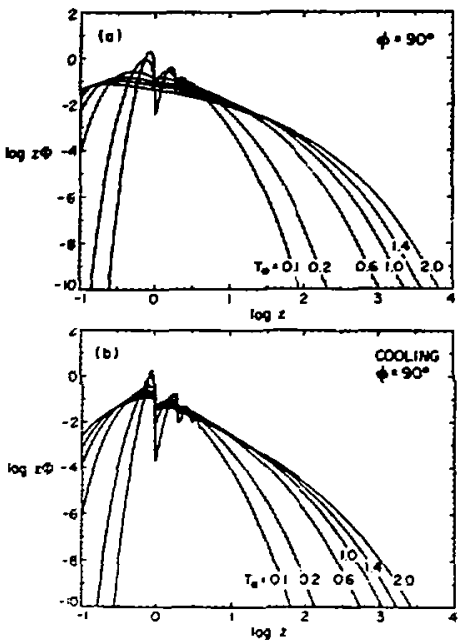

Fig. 8 Sample synchrotron emission spectra for thermal (a) and cooling (b) electron distributions (from Brainerd and Lamb 1987). 


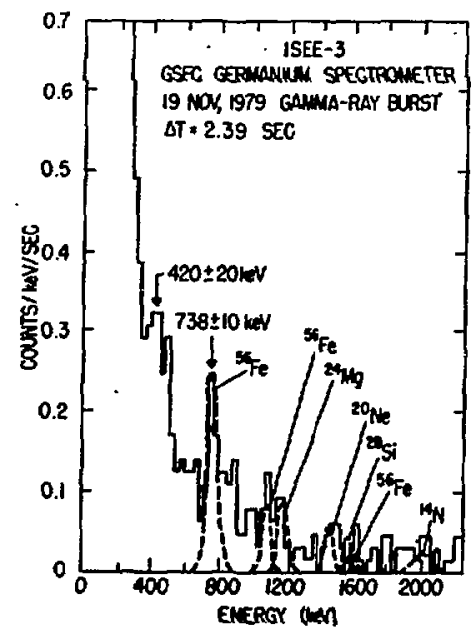

Fig. 9 Spectra of GB781119 showing emission features (from Teegarden and Cline 1980)

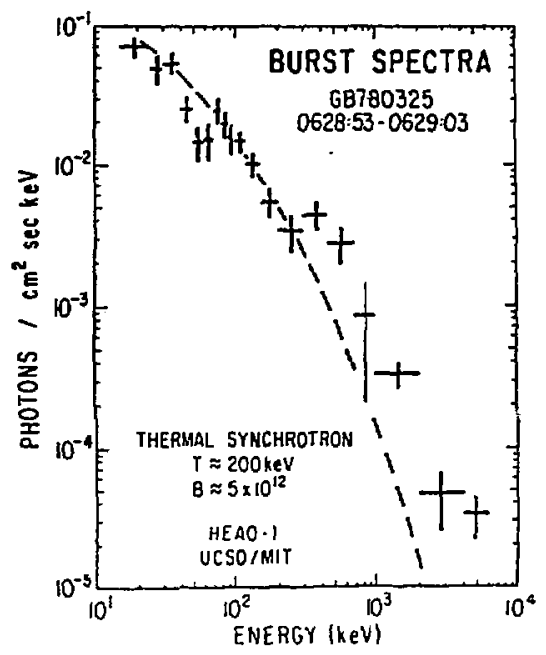

Fig. 10 HEAO-A4 spectra of GB780325 showing a braod bump at 400 $\mathrm{keV}$ (from Heuter 1987) 